\title{
Failed Intramedullary Screw Fixation of a Proximal Fifth Metatarsal Fracture (Jones Fracture) in a Division I Athlete: A case report
}

\author{
by Dane K. Wukich, MD $^{1 凹}$, Bora Rhim, DPM⿻上丨 , Dekarlos M. Dial, DPM ${ }^{3}$
}

The Foot and Ankle Online Journal 2 (6): 1

Intramedullary screw fixation of a Jones fracture is described in a basketball player (division I athlete). Early mobilization is the cornerstone to using intramedullary screw fixation in athletes. This case report describes the use of a smaller diameter screw to fixate a Jones fracture that failed. The authors have found that using a screw diameter similar to the diameter of the medullary canal may help to prevent screw failure.

Keywords: Intramedullary screw fixation, Jones fracture, screw failure.

Accepted: May, 2009

Published: June, 2009

This is an Open Access article distributed under the terms of the Creative Commons Attribution License. It permits unrestricted use, distribution, and reproduction in any medium, provided the original work is properly cited. OThe Foot and Ankle Online Journal (www.faoj.org)

$\int$ ones fracture of the fifth metatarsal has been defined as an acute fracture occurring in the proximal portion of the fifth metatarsal base at the metaphyseal and diaphyseal junction in which the fracture can involve the 4 th and $5^{\text {th }}$ intermetatarsal joints. ${ }^{1}$ Currently, there is no clear consensus on optimal treatment of acute Jones fractures in the athletic or the non-athletic population. There has been much debate in the treatment of proximal $5^{\text {th }}$ metatarsal fractures since described by Sir Robert Jones in 1902. ${ }^{2}$ The treatment of Jones fractures continues to remain controversial and challenging. ${ }^{3}$

\footnotetext{
Address correspondence to: Dekarlos M. Dial, DPM, Cornerstone Foot and Ankle Specialists, 1814 West Chester Drive, Suite 300, High Point, North Carolina 27262

${ }^{1}$ Chief, Foot and Ankle Division, Department of Orthopaedic Surgery; University of Pittsburgh Medical Center, Pittsburgh, Pennsylvania.

${ }^{2} 3$ rd year resident, Department of Graduate Medical Education; University of Pittsburgh Medical Center Surgery, Pittsburgh, Pennsylvania.

${ }^{3}$ Foot and Ankle Fellow, Department of Orthopaedic Surgery; University of Pittsburgh Medical Center, Pittsburgh, Pennsylvania.
}

Optimal screw selection for operative treatment in competitive athletes with $5^{\text {th }}$ metatarsal Jones fracture has not been determined. Cannulated screw fixation has been a popular method of fixation and has gained wide acceptance. Due to increased failure rates in elite athletes from refracture, delayed union, and non union, Wright, et al., recommended using a larger solid screw in competitive athletes to counter the higher amount of torsional stress placed on the fracture site. ${ }^{4}$ We present in this case report a Division I competitive basketball player who sustained a proximal $5^{\text {th }}$ metatarsal fracture. His initial treatment involved open reduction and internal fixation (ORIF) with a small diameter intramedullary screw. The patient developed a nonunion and required autogenous bone grafting with larger diameter screw fixation. Selection of screw type and diameter deserves thorough consideration. The authors utilize large diameter solid screws that are compatible with the $5^{\text {th }}$ metatarsal intramedullary canal diameter. 


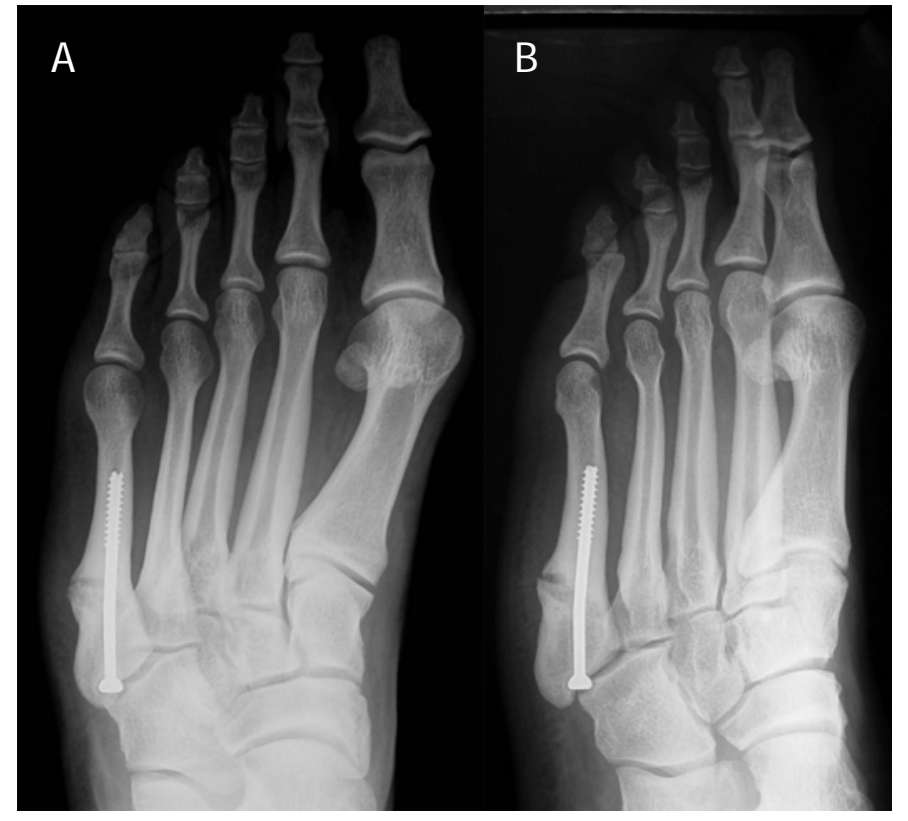

Figures 1A and 1B Anteroposterior and oblique radiographic views of the right foot demonstrating a bent $4.0 \mathrm{~mm}$ cannulated intramedullary screw. (A) The oblique radiograph demonstrates the nonunion of left proximal $5^{\text {th }}$ metatarsal fracture. (B)

\section{Case Report}

A 21-year-old male collegiate basketball player presents with right foot pain. His symptoms began after jumping and landing awkwardly. He developed severe pain on the lateral border of his right foot. The pain is exacerbated with weightbearing and walking. The patient also reports a similar injury involving the right foot that resulted in a $5^{\text {th }}$ metatarsal fracture 9 months prior.

The initial injury was treated operatively with intramedullary screw $(4.0 \mathrm{~mm}$ cannulated) fixation. He is $6.9 \mathrm{ft}$ tall, weighs $113 \mathrm{~kg}$ and in good health. The patient is not taking medications and denies any drug allergies. After the initial ORIF, he played in the 2004 - 2005 season. He reported intermittent pain of his right foot at the time.

The patient was evaluated after sustaining the second injury. He walked with an antalgic gait and had no deformity or atrophy.

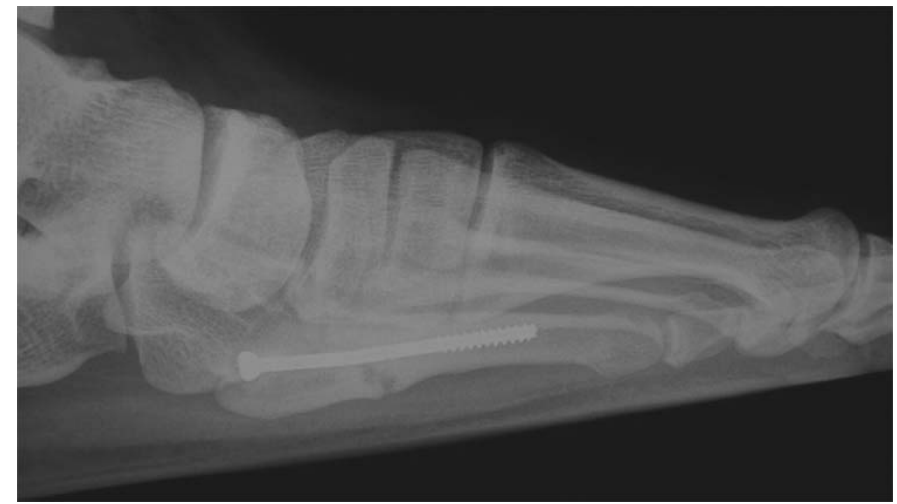

Figure 2 Pre-operative lateral radiograph right foot demonstrating nonunion of left $5^{\text {th }}$ metatarsal Jones fracture and bending of the screw fixation.

The foot was tender to palpation at the base of the $5^{\text {th }}$ metatarsal. Neurovascular status was intact with normal range of motion of his right foot and ankle.

Plain radiographs of the right foot revealed a nonunion of the proximal $5^{\text {th }}$ metatarsal right foot. There was a $4.0 \mathrm{~mm}$ intramedullary cannulated screw within the $5^{\text {th }}$ metatarsal. The screw was bent on both the anteroposterior and oblique radiographs. (Figs. $1 \mathrm{~A}$ and $\mathrm{B}$ ) The lateral preoperative views also show bending forces within the screw. (Fig. 2) A computed tomography (CT) scan was ordered and revealed a $50 \%$ incomplete union of a fifth metatarsal fracture consistent with nonunion. (Figs. 3A and B) The patient was then scheduled for hardware removal, bone grafting and screw exchange with a larger diameter intramedullary fixation screw. Before surgery, the patient was placed in a CAM walker with weight bearing to tolerance. In surgery, the $4.0 \mathrm{~mm}$ cannulated intramedullary screw was identified and removed. The nonunion site was identified and curettaged. Autogenous bone graft was harvested from the ipsilateral calcaneus. A larger diameter 6.5 $\mathrm{mm}$ screw was placed in the intramedullary canal to achieve appropriate stabilization. He was placed in a compressive dressing immediately postoperatively and transferred into a below-the-knee fiberglass cast on the fifth post-operative day. Clinical union was achieved at 6-weeks and the patient was then asymptomatic. He was advanced to protected weight bearing in a CAM walker. (Figs. 4A and B) 


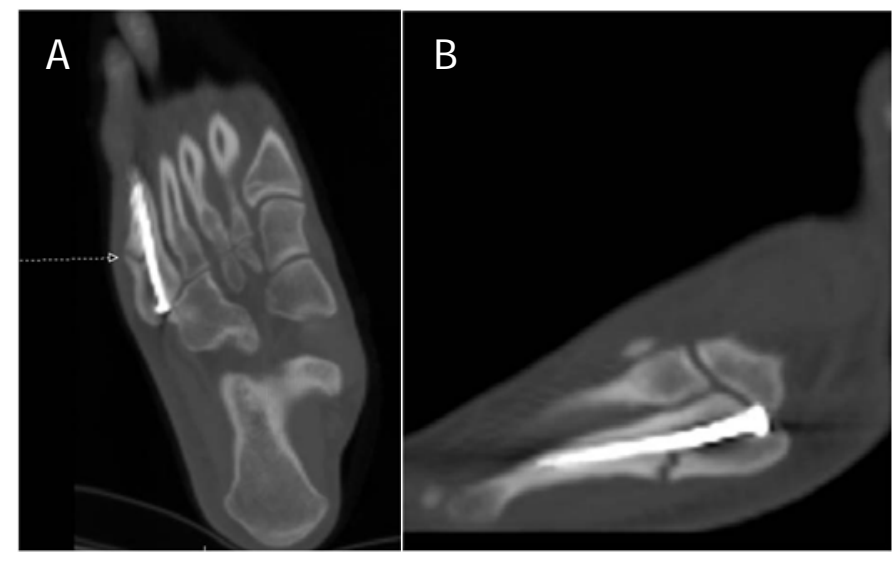

Figures 3A and 3B CT scan of the right foot demonstrating non-union of right proximal $5^{\text {th }}$ metatarsal (arrow).(A) Reformatted CT scan right foot demonstrating plantar lucency involving $50 \%$ of the proximal plantar cortex. (B)

At this time, stationary biking exercises were permitted. At 3-month follow-up, complete radiographic consolidation was noted at the fracture site. A CT scan was ordered and demonstrated complete radiographic healing of the fracture at 5 months. (Figs. 5A and B) The patient was permitted to return to competitive sports at 6 month follow-up.

\section{Discussion}

The type of screw fixation in the treatment of Jones fractures is controversial.. Many surgeons support intramedullary screw fixation for Jones Fractures. ${ }^{1,5,6,7,8,9}$ Kelly, et al., found that a $6.5 \mathrm{~mm}$ screw was superior to a $5.0 \mathrm{~mm}$ screw with respect to both pullout strength (in medullary canals greater than $5 \mathrm{~mm}$ ) and cantilever bending forces. ${ }^{6}$ Excessive repetitive cantilever forces applied to a suboptimal smaller diameter screw may result in bending and ultimately screw failure resulting delayed or nonunion. For this reason, utilizing a large diameter screw in the larger athletic patient population has been advocated. ${ }^{4,9,10}$ Vertullo, et al., encouraged utilizing an internal fixation device with the capability to resist torsion as well as bending. ${ }^{11}$

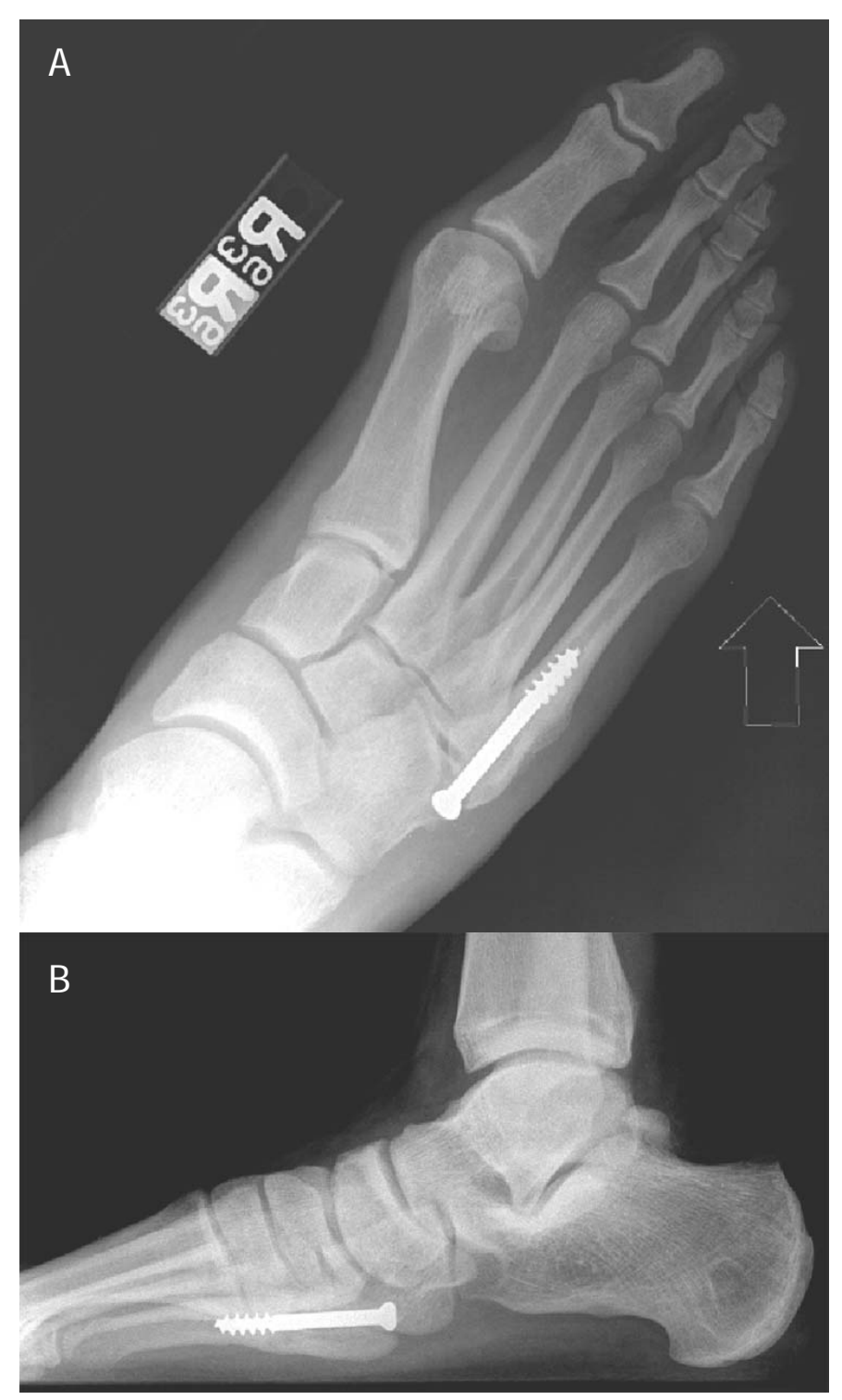

Figure 4A and 4B Post-operative anteroposterior radiograph left foot demonstrating radiographic union after revisional surgery with a $6.5 \mathrm{~mm}$ solid screw. (A) Post-operative lateral radiograph demonstrating complete radiographic union using a $6.5 \mathrm{~mm}$ solid screw along the plantar cortex with good alignment. (B) 

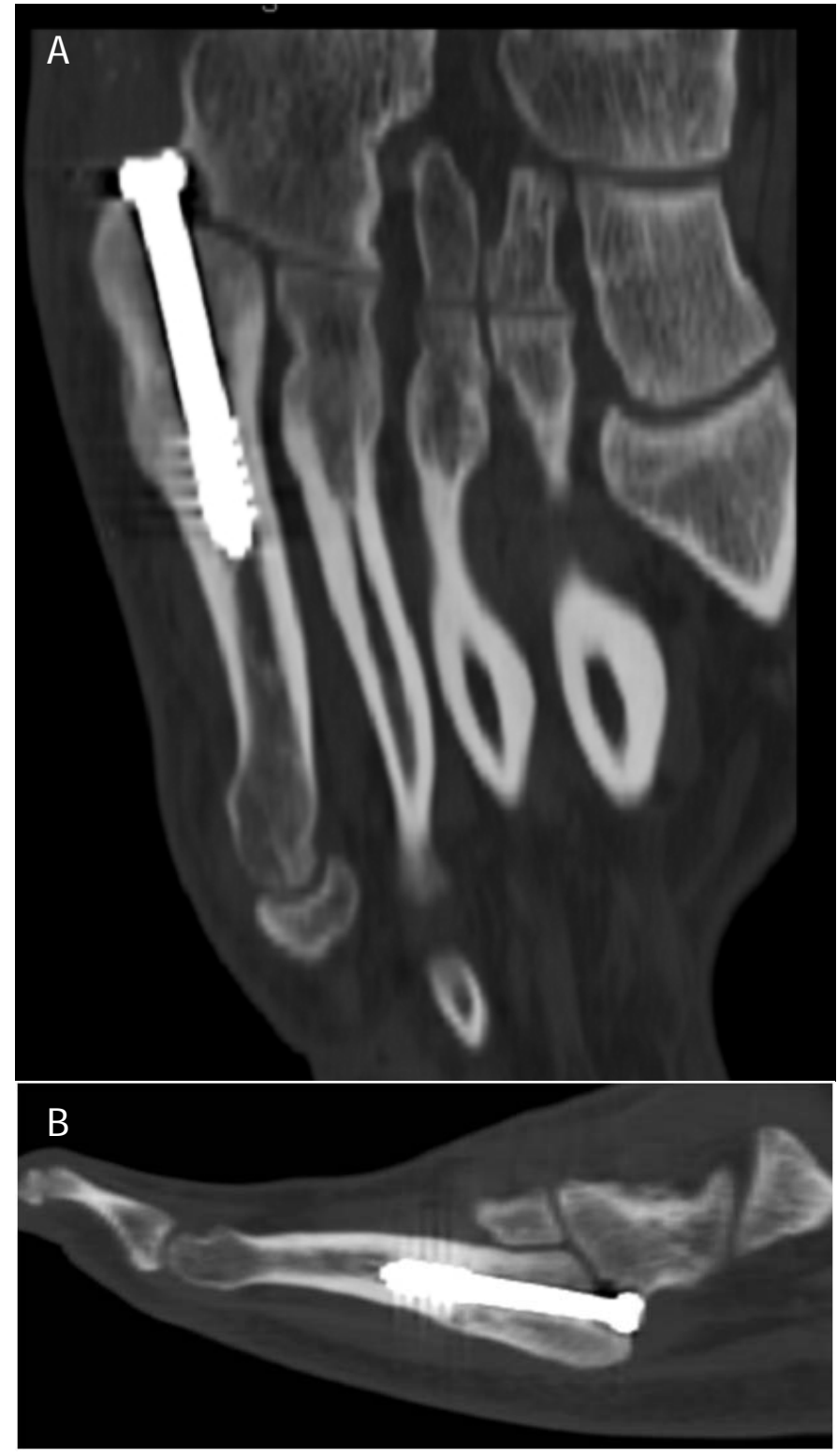

Figure 5A and 5B Post operative CT scan demonstrating stable $6.5 \mathrm{~mm}$ solid screw fixation with complete fracture union right $5^{\text {th }}$ proximal metatarsal. (A) Post operative reformatted sagittal CT scan. Note the stable fixation and complete fracture union. (B)

Refractures following initial intramedullary screw fixation of Jones fractures has been documented in athletes. Wright, et al., reported six refractures after complete radiographic and clinical union utilizing cannulated screw fixation of Jones fractures in athletes. $^{4}$
The refractures were attributed to insufficient screw diameter in athletes with a larger body mass and failure to incorporate functional bracing during first season of play.

Glasgow, et al., concluded that insufficient screw selection and vigorous return to activity appeared to correlate with failure and strongly discouraged intramedullary fixation with any device other than a 4.5 malleolar screw. ${ }^{9}$

Conversely, Porter, et al., reported on 23 consecutive athletes treated surgically with a 4.5 cannulated stainless steel screw for Jones fractures. ${ }^{12}$ The authors reported $100 \%$ clinical healing, mean radiographic healing rate of $98.9 \%$ and a zero incidence of refracture in this series. Larson et al reported a $40 \%$ (6 of 15) failure rate of patients treated with initial intramedullary screw fixation. ${ }^{10}$ There were a higher proportion of elite athletes (division I or professional level) among the failure group (83\%) compared with those without complications $(11 \%)$. None of the screws fractured in the failure group, but it was noted intraopratively that three were bent. There were no significant differences in age, sex, and screw diameter, use of bone graft or age of fracture between patients with failures and those without complications. In the current case report, suboptimal screw diameter was implicated as the precursor to refracture and failure.

Operative and non-operative treatment for Jones fractures has been described in the literature; however, in competitive athletic patients, operative treatment appears to be more favorable. ${ }^{13,14}$ Due to vascularity, muscle insertions, and motion related to the fifth metatarsal, O'Shea, et al., recommend that most Jones fractures be internally fixated for a more rapid return to function. ${ }^{15}$ Early operative treatments of acute Jones fractures results in quicker times to union and return to sports compared with cast treatment. ${ }^{5,13,15,16}$ Konkel, et al., recommended nonoperative treatment of fifth metatarsal fractures for patients in whom the time to full activities is not critical. $^{17}$ 
The fifth metatarsal shaft bowing and intramedullary canal width deserve special attention. If unrecognized, variations in $5^{\text {th }}$ metatarsal diaphyseal anatomy could lead to intraoperative morbidity. Ebraheim, et al., demonstrated that the intramedullary canal is bowed and the dorsoplantar diameter is more than $1 \mathrm{~mm}$ narrower than the mediolateral diameter. ${ }^{18}$

Pre-operative lateral and oblique radiographs allow assessment of severe lateral bowing of the shaft. ${ }^{3}$ We concur with Ebraheim, et al., in that the intramedullary canal assessment allows for precise and accurate screw placement.

When utilizing intramedullary screw fixation for Jones fractures, we interpose the screw over the metatarsal under fluoroscopy. This facilitates accurate intramedullary screw selection and avoids potential intraoperative fracture.

Zelko, et al., reported that athletes in sports such as football or soccer are often able to participate in sports while the fracture is healing and basketball players are most disabled and require surgical treatment. ${ }^{19}$ Kavanaugh, et al., noted a predilection for failure of varsity basketball players treated nonoperatively. ${ }^{13}$ In theory, the repetitive jumping and running of basketball increases cantilever bending at the fracture site compromising union.

Pietropaoli, et el., conducted a biomechanical study demonstrating no biomechanical difference between a $4.5 \mathrm{~mm}$ malleolar screw and a $4.5 \mathrm{~mm}$ partially threaded cancellous cannulated screw. ${ }^{20}$ The physiologic loading of bone may be greater in the high performance athlete with a larger body mass; making smaller screws vulnerable to bending. In the study by Wright, et al., all patients were athletes and returned to full-speed activity an average of 8.5 weeks post-fixation. ${ }^{4}$ Speculation on the cause of re-fracture included early return to activity, insufficient screw diameter, use of cannulated screws, and large patient body mass as possible sources. This case report is consistent with Wright's study and we believe larger diameter screws are required in patients with a larger body mass.
In conclusion, intramedullary screw fixation provides excellent stabilization in proximal $5^{\text {th }}$ metatarsal fractures. The $5^{\text {th }}$ metatarsal diaphyseal anatomy and patient body mass deserve thorough consideration in selecting a screw that affords adequate endosteal purchase and stability.

\section{References}

1. Nunley JA. Jones fracture technique. Techniques in Foot and Ankle Surgery 2: $131-137,2002$.

2. Jones R. Fracture of the base of the fifth metatarsal bone by indirect violence. Ann Surg 35: 697 - 700, 1902.

3. Horst F, Gilbert BJ, Glisson RR, James A: Torque resistance after fixation of Jones fractures with intramedullary screws. Foot \& Ankle Int 25 (12): 914 - 919, 2004.

4. Wright RW, Fischer DA, Shively RA, Heidt RS Jr, Nuber GW: Refracture of proximal fifth metatarsal (Jones) fracture after intramedullary screw fixation in athletes. Am J Sports Med 28 (5): $732-736,2000$.

5. DeLee JC, Evans JP, Julian J: Stress fractures of the fifth metatarsal. Am J Sports Med 11(5): 349 - 353, 1983.

6. Kelly IP, Glisson RR, Fink C, Easley ME, Nunley JA: Intramedullary screw fixation of Jones fractures. Foot Ankle Int 22 (7): $585-589,2001$.

7. Nunley JA. Fractures of the base of the fifth metatarsal: The Jones Fracture. Ortho Clin North Am 32: 171 - 180, 2001.

8. Shah SN, Knoblich GO, Lindsey DP, Kreshak J, Yerby SA, Chou LB: Intramedullary screw fixation of proximal fifth metatarsal fractures: a biomechanical study. Foot Ankle Int 22 (7): $581-584,2001$.

9. Glasgow, MT, Naranja, RJ, Glasgow SG, Torg JS: Analysis of failed surgical management of fractures of base of the fifth metatarsal distal to the tuberosity: The Jones fracture. Foot Ankle Int 17 (8): 449 - 457, 1996.

10. Larson C, Almekinders L, Taft T, Garrett, W: Intramedullary Screw Fixation of Jones Fractures: Analysis of Failure. Am J Sports Med 30: 55 - 60, 2002.

11. Vertullo C, Glisson R, Nunley J: Torsional Strains in the Proximal Fifth Metatarsal: Implication for Jones and Stress Fracture Management. Foot Ankle Int 25(9): 650 - 656, 2004.

12. Porter D, Duncan M, Meyer S: Fifth metatarsal Jones fracture fixation with a $4.5 \mathrm{~mm}$ cannulated stainless steel screw in the competitive and recreational athlete: A clinical and radiographic evaluation. Am J Sports Med 33(5): 726 - 733, 2005.

13. Dameron TB Jr: Fractures of the proximal fifth metatarsal: selecting the best treatment option. J Am Acad Orthop Surg 3: 110 - 114, 1995.

14. Kavanaugh JN, Brower TD, Mann RV: The Jones fracture revisited. J Bone Joint Surg 60A: 776 - 782, 1978. 
15. O'Shea MK, Spak W, Sant'Anna S, Johnson C: Clinical perspective of the treatment of $5^{\text {th }}$ metatarsal fractures. JAPMA 85 (9) :473 - 480, 1995.

16. Mologne T, Lundeen J, Clapper M, O’Brien T: Early Screw Fixation Versus Casting in Acute Jones Fractures. Am J Sports Med 33 (7): 970 - 975, 2005.

17. Konkel K, Menger A, Retxlaff S. Nonoperative treatment of fifth metatarsal fractures in an orthopaedic surburban private multispecialty practice. Foot Ankle Int 26(9): 704 - 707, 2001.

18. Ebraheim NA, Haman SP, Lu J, Padanilam TG, Yeasting RA. Anatomical and radiological considerations of the $5^{\text {th }}$ metatarsal bone. 21(3): Foot Ankle Int, 212 - 215, 2000.

19. Zelko RR, Torg JS, Rachum A: Proximal diaphyseal fractures of the fifth metatarsal (Jones) fracture after intramedullary screw fixation in athletes. Am J Sports Med 28: $732-736,2000$.

20. Pietropaoli MP, Wnorowski DC, Wener FW, et al. Intramedullary screw fixation of Jones fractures: A biomechanical study. Foot Ankle Int 20 (9): 560 - 563, 1999. 